\title{
Analysis of the Sagittal Motion Posture of the Acromioclavicular Joint Using Image Registration and Axial Angle Representation
}

\author{
Peng Su ${ }^{1,2}$ \\ Jun-lin Zhou ${ }^{2}$ \\ Cai Yun' \\ Feng Liu' \\ Yi Zhang'
}

'Department of Orthopaedics, Teaching Hospital of Capital Medical University, Beijing Shijingshan Hospital, Beijing, People's Republic of China; ${ }^{2}$ Department of Orthopedics, Beijing Chaoyang Hospital, Capital Medical University, Beijing, People's Republic of China
Correspondence: Jun-lin Zhou Department of Orthopedics, Beijing Chaoyang Hospital, Capital Medical University, 8 Gongren Tiyuchang Nanlu, Chaoyang District, Beijing, 100020,

People's Republic of China

Tel +86-132407/8193

Email zhoujunlin145@163.com
Objective: This study aimed to directly and accurately measure the range of motion of the acromioclavicular joint through 3D reconstruction and image registration.

Methods: Thirteen healthy volunteers participated in the study. Computerized tomography (CT) was used to measure the acromioclavicular joint in four different motion poses. The images were integrated using reconstruction and registration technology, and the rotation angle range of the acromioclavicular joint was measured using $3 \mathrm{D}$ reconstruction. The measurements were expressed by axial angle representation. The dominant and the nondominant sides were compared, and the difference in the axial angle of the acromioclavicular joint was compared in different postures.

Results: The difference between the dominant and non-dominant sides in acromioclavicular motion was not significant. In the sagittal motion of the upper limb, the rotation angle of the acromioclavicular joint was greatest in a resting horizontal position, with an average of $26.1^{\circ}$. In this position, $34.6 \%$ of flexion and extension movements of the upper limb were completed by the acromioclavicular joint, which was the highest level of engagement. The rotation angle was lowest in the resting posteroinferior position, with an average of $8.2^{\circ}$. In this position, $24.9 \%$ of the flexion and extension movements of the upper limb were completed by the acromioclavicular joint.

Conclusion: Combining 3D reconstruction and image registration is a direct and accurate method of measuring the motion of the acromioclavicular joint. The rotational motions of the acromioclavicular joint are bilaterally symmetrical and can be used as a reference for comparative study. The maximum range of motion of the acromioclavicular joint during rotation was found in the resting horizontal position. The clinical examination and post-treatment evaluation of the acromioclavicular joint's rotation function can therefore be targeted in this range.

Keywords: image registration, axial angle representation, acromioclavicular joints

\section{Introduction}

The acromioclavicular joint connects the clavicle with the scapula and forms a combined motion with the sternoclavicular joint and glenohumeral joint to complete the function of the shoulder girdle. ${ }^{1}$ It allows the scapula additional range of rotation on the thorax. The acromioclavicular joint is therefore an important part of synchronous combined motions. However, it is difficult to measure the joint's range of motion because it is affected by both clavicle and glenohumeral joint movements.

For anatomical reasons, the acromioclavicular joint has two forms of rotation and displacement. Codman and Rockwood ${ }^{1,2}$ both identified synchronous 
movements of the clavicle and scapula and found that the motion of the acromioclavicular joint is only $5-8^{\circ}$ when the shoulder is raised. A study by Inman $^{3}$ revealed that full shoulder elevation includes clavicle axial rotation of $40-50^{\circ}$, elevation of $11-15^{\circ}$, and rear extension of 15-30 . Sahara $^{4}$ found that, during the coracoid, the scapula usually rotates around a specific screw axis via the acromioclavicular ligament and the coracoclavicular ligament, with an average rotation of $34.9^{\circ}$.

In 2015, the International Society of Biomechanics (ISB) $)^{5}$ recommended using a joint coordinate system, bone marker points, and Euler angles to represent joint rotation. However, the clinical definition of Euler angles is not consistent in $3 \mathrm{D}$. Lawrence ${ }^{6}$ found that the rotation of the scapula is defined by three rotations of Euler angles. Her results showed that the motions of the acromioclavicular joint include an internal rotation of $60.0-62.3^{\circ}$, an upper rotation of $2.5-2.9^{\circ}$, and a forward inclination of $8.2-8.4^{\circ}$. The joint motion described by the ISB defines a rotation sequence (such as the Y-Z-Y of the Euler angle) so that the angle is as close to the clinical definition of joints and segmental motions as possible. However, the Euler angle cannot easily be used to describe the motion of the acromioclavicular joint, especially when the description of the joint's rotation does not follow clinical terminology. ${ }^{7}$

Following the standardized protocol for the recording of shoulder movement proposed by van der Helm, ${ }^{13}$ a 3D video (VICON and Optotrack, etc.) of the chest and humerus is usually recorded. However, clavicle and scapular movements cannot be recorded using external markers due to significant bone and skin displacements. Anglin ${ }^{7}$ stated that the rotations of the scapula and clavicle are difficult to track with external markers because of the large amount of movement under the skin. It is the most authentic and accurate way of measuring the angle between bones directly in vivo.

Axial angle representation is a method of describing joint rotation in $3 \mathrm{D}$ space. It defines the rotation of an object in 3D space with an axis or a straight line, describing the amount of rotation around the axis using an angle, thereby achieving a definition of the action that corresponds to a rotation axis and a rotation motion. This avoids the three rotation order problems of Euler angles. Because there is only one rotation, the interaction problem of subsequent rotation is also effectively avoided. In 1989, Sangeorzan ${ }^{8}$ stated that axial angle representation (rotation vector) was an intuitive way of describing rotation in $3 \mathrm{D}$ space and that it was suitable for clinical operation in the design of orthopedic surgery. $\mathrm{Wu}^{9}$ also used axial angle representation when studying the computer-aided design of orthopedic devices in 2014.

There are many ways to detect the movements between the 4 different positions and postures of the boom sections, ${ }^{7}$ including electromagnetic and sonic sensors, passive and active optical markers, an electric goniometer, a potentiometer-based beat-frequency detector, X-ray stereogrammetry, and magnetic resonance imaging (MRI). A significant problem with these methods, however, is the interference of respiratory movement and sternal position changes on measurements. Image registration solves this problem. The main principle of image registration is the correlation of spatial transformation between images, transforming the problem from a registration problem into a mathematical parameter optimization problem.

It is often necessary to convert images registered in different modes into images ${ }^{10}$ with the same mode. Lawrence's contrastive study ${ }^{11}$ revealed that a singleplane perspective and 2D/3D shape matching can accurately quantify the complex kinematics of a shoulder in a resting position, thereby proving that image registration technology can achieve the accuracy of a single-plane perspective. Seo ${ }^{12}$ studied the motion of the acromioclavicular joint in different movements between the 4 different positions and the changes in ligament length and tension using a $3 \mathrm{D}$ registration technique based on computerized tomography $(\mathrm{CT})$ images.

In the present study, in order to find a way to directly and accurately measure the range of motion of the acromioclavicular joint through $3 \mathrm{D}$ reconstruction and image registration, a $3 \mathrm{D}$ rotation of the scapula relative to the clavicle was used as a study object.

\section{Methods \\ Study Subjects}

A total of 13 healthy adults with no history of shoulder trauma or chronic pain were enrolled in this study. The sample was made up of 7 men and 6 women, with ages ranging from 22 to 42 and a mean age of 26.5 years. Their BMI scores were between 18.9 and 39.2, with a mean of 24.2. The dominant shoulder of 2 of the participants was their left, while for the other 11 it was their right.

This study was conducted with approval from the Ethics Committee of Beijing Shijingshan Hospital (201913). This study was conducted in accordance with the declaration of Helsinki. Written informed consent was obtained from all participants. 


\section{Image Acquisition}

CT scan (Discovery CT750HD, GE). Parameter settingsSlice thickness: $0.625 \mathrm{~mm}$; layer distance: $0.625 \mathrm{~mm}$. Shooting position - (a) Supine and resting position (the baseline position which the three other movements were compared to); (b) supine position with forward extension of the elbow joint, horizontal position of upper limb; (c) prone position with excessive posterosuperior extension of the elbow joint, posterosuperior position of upper limb; (d) prone position with back extension of the elbow joint, posteroinferior position of upper limb. Shooting rangeFrom $2 \mathrm{~cm}$ below the sternal angle plane to the extremitas acromialis claviculae, including the proximal humerus above the deltoid tuberosity. See Figure 1. In this experiment, healthy volunteers were examined by CT for four times, and the cumulative radiation dose was as follows: Minimum value: 570.15 Maximum value: 1950.84 Mean 1231.5777 \pm 449.96090 DLP(MGY-CM).

\section{Image Registration}

Raw image data were imported into MIMICS 21 software to form a 3D image complex with four postures containing the sternum, clavicle, and humerus. In accordance with ISB recommendations, a thoracic reference coordinate system and bone reference points were adopted. The motion of the acromioclavicular joint was considered the rotation of the scapula relative to the clavicle represented by axial angle. The 3D images of the sternum in a supine and resting position were used as registration objects to register the 3D image complex in other poses. Finally, the images of the clavicle in four different postures were overlapped, and the 3D images of the scapula and humerus were registered in different poses. See Figure 2 and Supplemental Figure 1.

\section{Data Acquisition and Processing}

The participants were divided into two groups according to their dominant shoulders, then into three groups according to posture changes (resting horizontal, horizontal posterosuperior, and resting posteroinferior). Using the baseline 3D image complex of the clavicle, scapula, and humerus in a supine and resting position, the rotation angle of the acromioclavicular joint in different postures was measured and expressed as an axial angle. The corresponding flexion and extension angle of the humerus was measured.

\section{Statistical Methods}

An independent sample $t$-test was used to compare the data of the two groups (left-side dominant and right-side

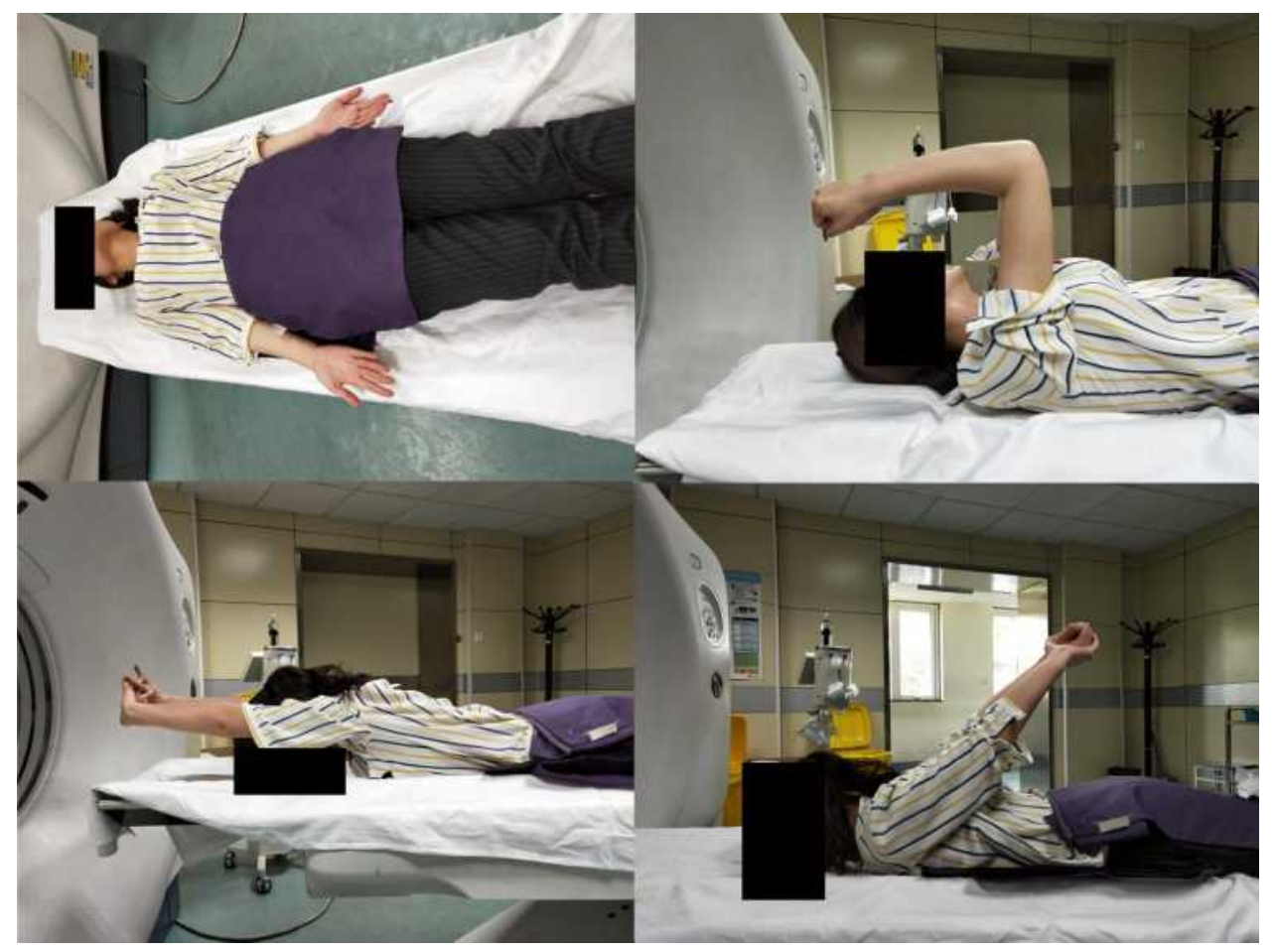

Figure I Image shooting movements between the 4 different positions: I) resting position, 2) $90^{\circ}$ of anterior flexion, 3) complete anterior flexion, and 4) complete posterior extension of the shoulder. 


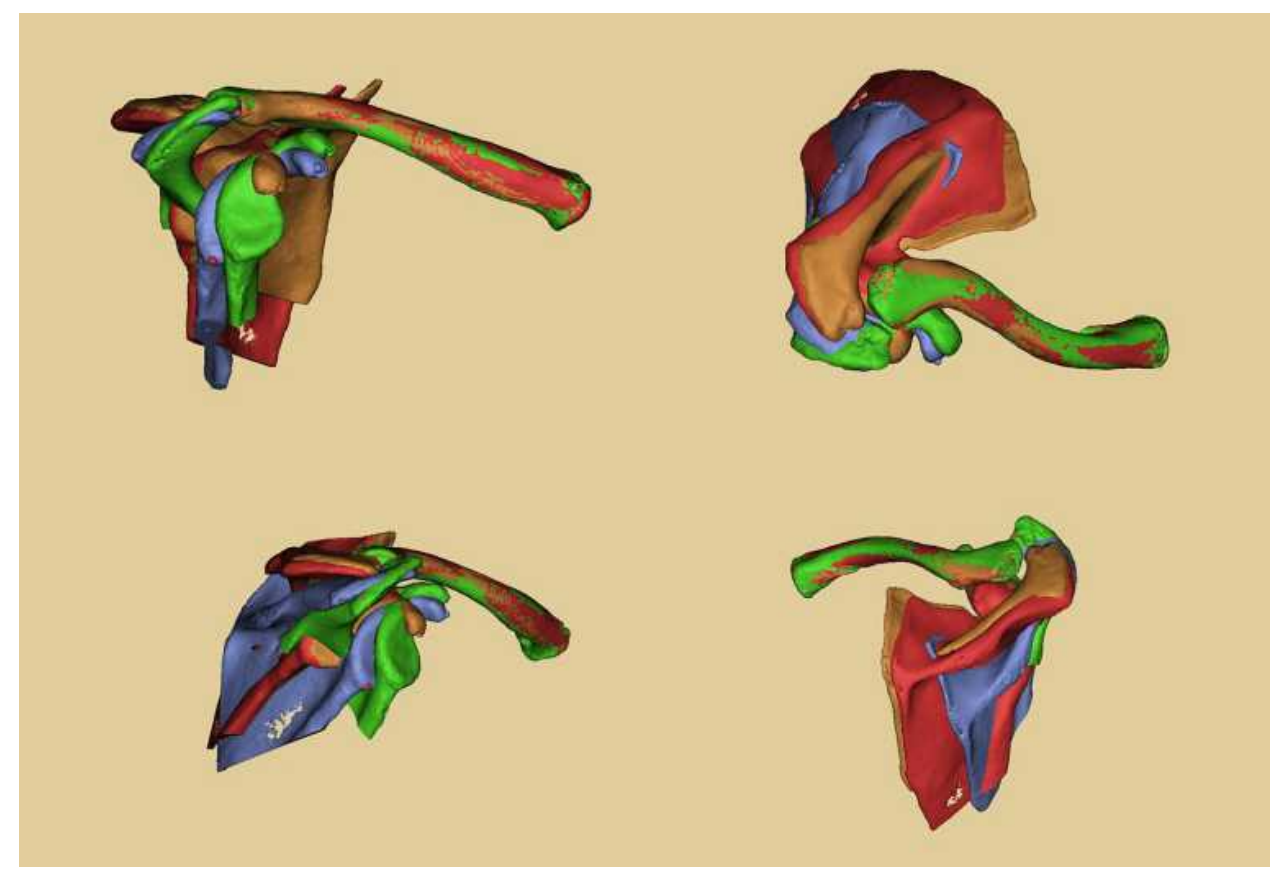

Figure 2 3D images after registration: front view, vertical view, side view, and rear view.

dominant) in three different posture angles. $P<0.05$ was considered statistically significant.

The three groups of data from the humerus and acromioclavicular joint in three different postures were processed to calculate the axial angles of the acromioclavicular joint in different poses. Grouping was then carried out based on posture. Statistical analysis was conducted using analysis of variance (ANOVA). $P<$ 0.05 was considered statistically significant. The contribution of the acromioclavicular joint's angle of motion to brachial axillary joint motion range was grouped into different poses (acromioclavicular joints/thoracohumeral joints), and statistical analysis was carried out using a chi-squared test. SPSS 19 software was used for statistical analysis.

\section{Results}

\section{Comparison of the Acromioclavicular} Joint Angle and Humeral Angle in Different Postures Between Dominant and Non-Dominant Sides in Sagittal

\section{Motion $(n=13)$}

The axial angle of the acromioclavicular joint, humeral flexion, and extension angle in different postures in the dominant and non-dominant sides were compared. See Table 1.

\section{Statistical Description of the Axial Angle of the Acromioclavicular Joint, Humeral Flexion, and Extension Angle in Different Postures in Sagittal Motion in 13 Patients (Table 2)}

The results of the chi-squared test, showing the contribution of the acromioclavicular joint (axial angle of acromioclavicular angle/humeral flexion and extension angle) are shown in Table 3.

Analysis of results: In the sagittal motion of the upper limb, the rotation angle of the acromioclavicular joint is greatest in a resting horizontal position, with an average of $26.1^{\circ}$. In this position, $34.6 \%$ of the flexion and extension motions of the upper limb are completed by the acromioclavicular joint, accounting for the greatest proportion of motions. The rotation angle is smallest in a resting posteroinferior position, with an average of $8.2^{\circ}$. In this position, $24.9 \%$ of the flexion and extension motions of the upper limb are completed by the acromioclavicular joint.

\section{Discussion}

\section{The Advantage of 3D Image Registration Techniques}

Although Seo ${ }^{12}$ conducted studies using image registration techniques, no $3 \mathrm{D}$ image registration techniques were 
Table I Comparison of Axial Angle of Acromioclavicular Joints and Humeral Flexion and Extension Angle Between the Dominant Side and Non-Dominant Side Under Different Postures

\begin{tabular}{|c|c|c|c|c|c|}
\hline & Postures & & Mean \pm SD & T-value & Sig \\
\hline Axial angle of acromioclavicular joints & $\begin{array}{l}\text { Resting - horizontal } \\
\text { Horizontal-posterosuperior } \\
\text { Resting- posteroinferior }\end{array}$ & $\begin{array}{l}\text { Non-dominant sides } \\
\text { Dominant sides } \\
\text { Non-dominant sides } \\
\text { Dominant sides } \\
\text { Non-dominant sides } \\
\text { Dominant sides }\end{array}$ & $\begin{array}{l}25.9208 \pm 7.97070 \\
26.2008 \pm 5.4603 \\
\mid 9.5392 \pm 6.6448 \\
|8.8462 \pm 6.7| 7 \mid \\
8.733 \mid \pm 3.8766 \\
7.7038 \pm 4.4|4|\end{array}$ & $\begin{array}{l}2.6 \\
0.06 \\
0.5\end{array}$ & $\begin{array}{l}0.7 \\
0.8 \\
0.5\end{array}$ \\
\hline Humeral flexion and extension angle & $\begin{array}{l}\text { Resting - horizontal } \\
\text { Horizontal-posterosuperior } \\
\text { Resting- posteroinferior }\end{array}$ & $\begin{array}{l}\text { Non-dominant sides } \\
\text { Dominant sides } \\
\text { Non-dominant sides } \\
\text { Dominant sides } \\
\text { Non-dominant sides } \\
\text { Dominant sides }\end{array}$ & $\begin{array}{l}83.4705 \pm 13.9086 \\
81.3761 \pm 12.7083 \\
96.2585 \pm 13.8511 \\
99.2588 \pm 16.4926 \\
47.4823 \pm 11.5488 \\
48.2270 \pm 10.1962\end{array}$ & $\begin{array}{l}0.3 \\
0.2 \\
0.2\end{array}$ & $\begin{array}{l}0.6 \\
0.7 \\
0.7\end{array}$ \\
\hline
\end{tabular}

Table 2 Statistical Description of Axial Angle of Acromioclavicular Joints/Humeral Flexion and Extension Angle Under Different Postures

\begin{tabular}{|c|c|c|c|c|c|c|}
\hline \multirow[t]{2}{*}{ Postures } & \multirow{2}{*}{$\begin{array}{l}\text { Axial Angle of Acromioclavicular } \\
\text { Joints } \\
\text { Mean } \pm \text { SD }\end{array}$} & \multicolumn{2}{|c|}{ ANOVA } & \multirow{2}{*}{$\begin{array}{l}\text { Humeral Flexion and Extension } \\
\text { Angle } \\
\text { Mean } \pm \text { SD }\end{array}$} & \multicolumn{2}{|c|}{ ANOVA } \\
\hline & & $\mathbf{F}$ & Sig & & $\mathbf{F}$ & Sig \\
\hline I:Resting - horizontal & $26.0 \pm 6.7$ & 60.4 & 0.000 & $82.4 \pm \mid 3.1$ & 99.8 & 0.000 \\
\hline 2:Horizontal-posterosuperior & $19.2 \pm 6.6$ & & & $97.8 \pm 15.0$ & & \\
\hline 3:Resting- posteroinferior & $8.2 \pm 4.1$ & & & $47.8 \pm 10.7$ & & \\
\hline
\end{tabular}

Abbreviation: ANOVA, analysis of variance.

Table 3 Chi-Square Test for Axial Angle of Acromioclavicular Joints/Humeral Flexion and Extension Angle Under Different Postures

\begin{tabular}{|l|l|l|l|}
\hline Postures & Mean \pm SD & $\chi^{2}$ & Sig \\
\hline I:Resting - horizontal & $34.6 \pm 9.0$ & 234.7 & 0.000 \\
2:Horizontal-posterosuperior & $22.7 \pm 7.6$ & & \\
3:Resting- posteroinferior & $24.9 \pm 11.6$ & & \\
\hline
\end{tabular}

used. In the present study, the acromioclavicular joints of a healthy person were photographed via $\mathrm{CT}$, and the $3 \mathrm{D}$ images were reconstructed by computer. In the virtual 3D space, the acromioclavicular joints in different postures were uniformly registered to the sternum in a resting position, and the angle change of the acromioclavicular joints in different postures were measured directly in the $3 \mathrm{D}$ space. The advantage of this image registration technique is that it can effectively eliminate the interference of acromioclavicular motion, respiratory motion, and sternal motion, making the measurements more reliable. The changes in the angles of the acromioclavicular joints were also measured directly in the virtual 3D space using the $3 \mathrm{D}$ reconstruction technology of $\mathrm{CT}$, which is more accurate and objective.

\section{Euler Angle Representation and Axis Angle Representation}

Euler angle representation and axis angle representation are effective ways of describing the rotation of objects in 3D space. However, there are two problems in the description of Euler angles: ${ }^{14}$ order and interaction. The rotation order of Euler angles is defined by the individual, and each rotation order will have different angles. Hence, the ISB has suggested that the order of rotation is limited if Euler angles are used to represent rotation. Moreover, the Euler angle divides a motion into three rotating movements in different coaxial directions, which is inconsistent with the characteristics of human joint motions. Anglin ${ }^{7}$ stated that there are many different terms because these rotations do not follow clinical linguistics. For example, the elevation plane may not be the plane on which the action takes place, but merely the mathematical rotation 
required to define a particular resting position. Matsuki ${ }^{15}$ used the Euler angle to describe the comparison between rotations in the dominant shoulder and non-dominant shoulder. The study found differences in two of the three rotations. Lawrence $^{6}$ stated that the clinical significance of using the Euler angle to describe the rotation of different movements of the same joint is also difficult to understand.

Axial angle representation is a way of expressing rotations in $3 \mathrm{D}$ space, and is more accurate than description on a 2D plane. Compared with Euler angles, axial angle representation simplifies the description of joint rotations and makes them more intuitive and easier to compare. Jehee $^{16}$ showed that there is no singularity in $3 \mathrm{D}$ space and that axis angle representation (rotation vector) can parameterize rotations. Therefore, it is suggested that rotation should be represented by rotation vector. A description of the rotation of an independent joint in a composite motion is especially suitable for the shoulders.

Axial angle representation also effectively avoids the order problem and mutual interference problem of acromioclavicular joints described by Euler angles. In human multijoint composite motions, each joint motion tries to complete a certain action as simply as possible (with minimum energy consumption). Axis angle representation expresses the position change through one rotation, while Euler angle representation expresses the position change through three rotations. Therefore, axial angle representation is more in line with the movement characteristics of human anatomy and more closely reflects the characteristics of joint movement. As such, it is more useful as a guide for clinical practice.

\section{Sagittal Motion of the Upper Limb}

The results of this study show that differences between dominant and non-dominant sides in the 3D rotation angles of the acromioclavicular joint in the sagittal motion of the upper limb are not significant. The rotational motions of the acromioclavicular joint could be bilaterally symmetrical and may be used as a reference for comparative study. This provides a theoretical basis for clinical examination and post-treatment evaluation.

In the sagittal motion of the upper limb, the acromioclavicular joint has the greatest rotation angle and participation in the resting horizontal position; it has the smallest rotation angle in the resting posteroinferior position. This shows that the maximum action range of the acromioclavicular joint rotation is the resting horizontal position, and the clinical examination and post-treatment evaluation of the rotation function of the acromioclavicular joint can be targeted in this range.

\section{Conclusion}

The combination of 3D reconstruction and image registration can effectively reduce the interference of respiratory and sternal motion on measurements. It is a direct and accurate method for the measurement of acromioclavicular joint motions. Axial angle representation also simplifies the presentation of joint rotation, which makes it more intuitive, easier to compare, and more in line with the movement characteristics of human anatomy; therefore, it more closely reflects the characteristics of joint movement and is useful for the instruction of clinical practice. The rotational motions of the acromioclavicular joint are bilaterally symmetrical and can be used as a reference for comparative study. The maximum range of rotational motion of the acromioclavicular joint is in the resting horizontal position.

The study has two limitations: firstly, the sample size was small; secondly, radiographic tests were used, although the radiation dose is not very large. Further supplementary experiments can be carried out by increasing the sample size. Also, we did not measure the "in between" instant; we measured 4 positions, not a continuum.

\section{Acknowledgments}

We would like to acknowledge the hard and dedicated work of all the staff that implemented the intervention and evaluation components of the study.

\section{Funding}

This work was supported by the Scientific research project of key medical disciplines in Shijingshan District, Beijing (2018). The funding agency had no role in the study design; in the collection, analysis and interpretation of data; in the writing of the report; or in the decision to submit the article for publication.

\section{Disclosure}

The authors declare that they have no competing interests.

\section{References}

1. Codman EA. The Shoulder, Rupture of the Supraspinatus Tendon and Other Lesions in or About the Subacromial Bursa. Thomas Todd; 1934.

2. Rockwood CA Jr. Injuries to the acromioclavicular joint. $J$ Bone Joint Surg. 1944;26:1-30. 
3. Inman VT, Saunders JB, Abbott LC. Observations on the function of the shoulder joint. Clin Orthop Relat Res. 1996;330:3-12. doi:10.1097/00003086-199609000-00002

4. Sahara W, Sugamoto K, Murai M, Tanaka H, Yoshikawa H. 3D kinematic analysis of the acromioclavicular joint during arm abduction using vertically open MRI. J Orthop Res. 2006;24 (9):1823-1831. doi:10.1002/jor.20208

5. Wu G, van der Helm FC, Veeger HE, et al; International Society of Biomechanics. ISB recommendation on definitions of joint coordinate systems of various joints for the reporting of human joint motion-part II: shoulder, elbow, wrist and hand. $J$ Biomech. 2005;38(5):981-992. doi:10.1016/j.jbiomech.2004.05.042

6. Lawrence RL, Braman JP, Laprade RF, Ludewig PM. Comparison of 3-dimensional shoulder complex kinematics in individuals with and without shoulder pain, part 1_ sternoclavicular, acromioclavicular, and scapulothoracic joints. J Orthop Sports Phys Ther. 2014;44 (9):636-645. doi:10.2519/jospt.2014.5339

7. Anglin C, Wyss UP. Review of arm motion analyses. Proc Inst Mech Eng H. 2000;214(5):541-555. doi:10.1243/0954411001535570

8. Sangeorzan BP, Judd RP, Sangeorzan BJ. Mathematical analysis of single-cut osteotomy for complex long bone deformity. J Biomech. 1989;22(11-12):1271-1278. doi:10.1016/0021-9290(89)90230-3

9. Wu YY, Plakseychuk A, Shimada K. Computer-aided surgical planner for a new bone deformity correction device using axis-angle representation. Med Eng Phys. 2014;36(11):1536-1542. doi:10. 1016/j.medengphy.2014.08.006
10. Song G, Han J, Zhao Y, Wang Z, Du H. A review on medical image registration as an optimization problem. Curr Med Imaging Rev. 2017;13(3):274-283. doi:10.2174/1573405612666160920123955

11. Lawrence RL, Ellingson AM, Ludewig PM. Validation of single-plane fluoroscopy and 2D/3D shape-matching for quantifying shoulder complex kinematics. Med Eng Phys. 2018;52:69-75. doi:10.1016/j.medengphy.2017.11.005

12. Seo YJ, Yoo YS, Noh KC, et al. Dynamic function of coracoclavicular ligament at different shoulder abduction angles_a study using a 3-dimensional finite element model. Arthroscopy. 2012;28 (6):778-787. doi:10.1016/j.arthro.2012.04.001

13. van der Helm FCT. A standardized protocol for motion recordings of the shoulder. First Conference of the International Shoulder Group. 1997.

14. van der Helm FC, Pronk GM. Three-dimensional recording and description of motions of the shoulder mechanism. J Biomech Eng. 1995;117(1):27-40. doi:10.1115/1.2792267

15. Matsuki $\mathrm{K}$, Matsuki $\mathrm{KO}, \mathrm{Mu} \mathrm{S}$, et al. In vivo 3D analysis of clavicular kinematics during scapular plane abduction: comparison of dominant and non-dominant shoulders. Gait Posture. 2014;39 (1):625-627. doi:10.1016/j.gaitpost.2013.06.021

16. Lee J. Representing rotations and orientations in geometric computing. IEEE Comput Graph Appl. 2008;28(2):75-83. doi:10.1109/MCG.2008.37
International Journal of General Medicine

\section{Publish your work in this journal}

The International Journal of General Medicine is an international, peer-reviewed open-access journal that focuses on general and internal medicine, pathogenesis, epidemiology, diagnosis, monitoring and treatment protocols. The journal is characterized by the rapid reporting of reviews, original research and clinical studies across all disease areas. The manuscript management system is completely online and includes a very quick and fair peer-review system, which is all easy to use. Visit http://www.dovepress.com/ testimonials.php to read real quotes from published authors. 